\title{
HYPOTHESIS
}

\section{Role of cytological analysis of synovial fluid in diagnosis and research}

\author{
A J Freemont
}

The hypothesis I wish to examine is:

Because the cells in synovial fluid of diseased joints represent a tissue infiltrate that both reflects and contributes to the internal microenvironment of the joint, changes in the type and number of synovial fluid cells can be used to distinguish between joint diseases and investigate their pathogenesis.

Synovial joints are generally considered to consist of a number of discrete elements (capsule, cartilaginous articular surfaces, synovial fluid, and synovium ${ }^{1}$ ); and synovial fluid is regarded as a body fluid lying within a tissue space bounded by synovium. Because this arrangement is reminiscent of the major body cavities, synovium and synovial fluid are often discussed in similar terms to those used for the lining and contents of other body cavities. By analogy synovial fluid and the changes that it undergoes in disease are often likened to those seen in other body fluids; for instance, by the use of the term joint effusion. This almost subconscious view of the synovial joint has important implications for the way in which joints and joint diseases are perceived and, in particular, how changes in the amount and composition of synovial fluid are evaluated.

The synovial joint is so commonly regarded as containing a body cavity that it is perhaps surprising to discover that if its anatomy is studied in detail its structure is not in any way comparable with that of the major body cavities. The fundamental biological difference between the 'joint space' and the body cavities lies in the nature of the interface between the elements lining the 'joint space' (synovium and cartilage) and the synovial fluid.

It is accepted that cartilage consists of cells in a solid matrix with no discrete surface cell layer. ${ }^{2}$ Direct exchange of water, solutes, and certain macromolecules occurs between the synovial fluid and cartilage matrix. ${ }^{3}$ It is also well recognised that at the ultrastructural level the interface between cartilage and synovial fluid is ill defined. ${ }^{4}$ Thus at both the physical and chemical level there is no discrete boundary between cartilage and synovial fluid, there being a small but important area of the joint in which components of both are mixed.

Although the surface synoviocyte layer often appears as a discrete boundary zone between the liquid synovial fluid and the more solid synovium, electron microscopy shows that synoviocytes are structurally dissimilar to the mesothelial cells of pericardium, peritoneum, or pleura, and morphological ${ }^{4}$ and epitope expression studies ${ }^{5}$ have shown that in lineage and function synoviocytes and mesothelial cells are quite distinct. Although the synoviocytes that aggregate on the synovial side of the interface between 'solid' synovium and 'liquid' synovial fluid are surrounded by basement membranelike material, this is not a true basal lamina as it lacks the ordered trilaminar arrangement typical of this structure. ${ }^{6}$ Indeed, synoviocytes are not natural lining cells but are rather a mixture of bone marrow derived cells of the monocyte-macrophage lineage and locally derived secretory cells. ${ }^{8}$ Because in normal joints both the cell layer and the basement membrane-like material are incomplete, ${ }^{9}$ in places synovial fluid is in direct continuity with the extracellular fluid of the synovium. ${ }^{10}$ Thus there is not the cell layer and complex basal lamina characteristic of the lining of other body cavities that directly controls the exchange of fluid, ions, and macromolecules between conventional lining tissues and the adjacent tissue space. As a result the synovial intima is a relatively ineffectual barrier to the passage of fluid, macromolecules, and cells. ${ }^{8}$

The morphological relation between the apparently discrete elements within the joint is, as is indicated above, quite unlike the structure of any conventional body cavity. As might be predicted the synthesis of synovial fluid is also very different from the production of body cavity fluids. Unlike pleural or pericardial fluid, which is a highly selected residuum of predominantly low molecular weight components of tissue fluid, synovial fluid consists of a mixture of a filtrate of plasma and macromolecules produced by cells within adjacent tissue. ${ }^{11}$ The difference can be fully explained by the lack in the joint of any form of the cell/basement membrane 'filter' mechanism seen at the interface between fluid and tissue in the body cavities. When comparison is made with other body constituents it is found that in general terms there is no essential difference between the formation of synovial fluid and an extracellular tissue matrix. In view of the way in which it forms and its physical and chemical relationships with surrounding elements it is probably better, therefore, to regard synovial fluid, not as a carefully controlled transudate into a body cavity, but as a peculiarly liquid connective tissue. 
The absence of distinct boundary zones, the relatively free flow of water, solutes, and macromolecules between the component parts of the joint, and the functional interdependence of cartilage, synovium, and synovial fluid mean that the various elements of the joint behave as a continuum; effectively as a single tissue. It is true that for a single tissue the joint shows an unusual degree of heterogeneity, ranging from cellular, often fatty, synovium through paucicellular cartilage with a viscoelastic matrix to a virtually acellular liquid medium that represents a connective tissue stroma of a distinctive and unusually 'runny' kind; but this degree of heterogeneity is by no means unique to the synovial joint. Normal tissues, such as the intervertebral disc, ${ }^{12}$ are just as variable in the consistency, physical and chemical properties of their component parts, and lesions such as ganglia $^{13}$ and adventitial bursae ${ }^{14}$ represent pathological entities in which a similar degree of physical heterogeneity occurs within a single tissue.

It would be naive to believe that the heterogeneity of the various elements of the joint was limited to their physical properties. The obvious variation in consistency, cellularity, and vascularity are all pointers towards equally fundamental biochemical differences. The chemical, physical, and structural characteristics of each element of the joint are presumably important to their normal function ${ }^{11}$ and, in addition, they will affect the way in which each component responds to abnormal chemical and physical stimuli. ${ }^{15} 16$ This is the single most important consideration when assessing the significance of joint changes in disease.

Normal synovial fluid, like cartilage, is avascular and contains very few cells. ${ }^{17}$ The two innate cell types within normal synovial fluid are identical to the two types of synoviocytes that congregate at the interface between the synovium and the fluid. ${ }^{18}$ One group of these cells is phagocytic, ${ }^{19}$ the other group is synthetic, the products being important chemical constituents of the fluid. ${ }^{8}$ It seems probable that when present, both in the synoviocyte layer and within the fluid, these cells help to maintain the chemical and structural integrity of the synovial fluid tissue. Like nearly all tissues normal synovial fluid also contains migratory cells of the immune system-notably, lymphocytes and macrophages. ${ }^{20}$

In disease the number of cells changes as does the nature of the matrix. ${ }^{20}$ Invariably the total number of cells within the fluid component of the joint increases above normal, though in some so called 'non-inflammatory' disorders the concentration of cells may not change significantly because the volume of synovial fluid increases proportionally-that is the tissue becomes oedematous. In all joint diseases there is an inevitable increase in the number of synoviocyte-like cells in the synovial fluid, a process that parallels the increase in cell numbers in the synoviocyte layer. ${ }^{21}$ In the noninflammatory arthropathies such as osteoarthritis the increase in the number of synoviocyte-like cells represents the predominant change in cell numbers. In inflammatory arthritides the increase may be dramatic with the total cell number increasing up to one thousandfold and a marked change occurring in the proportions of the various cell types ${ }^{22}$ with an overwhelming increase in the number of blood derived leucocytes.

If as suggested above the synovial fluid is a specialised connective tissue then in inflammatory arthropathies the cells within it represent an inflammatory cell infiltrate. The number, type, and activity of these cells would then be subject to the same influences as in any other inflammatory infiltrate. These represent a complex series of chemical interactions between the local tissue matrix, native cells, inflammatory cells, blood products, and factors produced in adjacent tissues. As in other tissues the nature of the cellular response will vary with the initiating event and certain key properties of the tissue such as cellularity, vascularity, and the chemical composition of the intercellular matrix. The local (and generalised) effects of the inflammation would also be subject to the same influences as those operating in other tissues and as such are an inevitable consequence of the cell-cell and cell-matrix interactions induced by the chemical mediators of the inflammatory response. Inevitably the scope of these reactions will also be dependent upon the cellular and extracellular composition of the tissue. In other words the pattern of the cellular infiltrate in the synovial fluid and the subsequent changes in the fluid and adjacent tissues are a direct consequence of the nature of the inflammatory stimulus and the way in which the body responds to that stimulus within the limitations imposed by the local environment.

The important part played by the tissue in modifying the cellular inflammatory response to a stimulus is nowhere illustrated better than in synovial joints. Were the joint a uniform structure one might expect the cellular response to the inflammatory stimulus to be the same throughout. During times of intra-articular inflammation, however, the distribution of inflammatory cells within the various elements of the joint is very different. Usually the cartilage does not contain an inflammatory cell infiltrate of any sort, the synovium contains a mixed infiltrate in which lymphocytes and, to a lesser extent, plasma cells predominate, and the fluid contains a mixed inflammatory cell infiltrate in which the predominant cell is the polymorph. ${ }^{23}$ As it seems unlikely that there would be more than one trigger of inflammation in any one joint the diversity of the inflammatory cell infiltrates in different parts of the joint must be the result of a modification of the response by the local composition of the tissue.

If the inflammatory reaction within the joint was dependent upon both the nature of the tissue and the type of stimulus one would predict that not only would the cellular infiltrate vary in different parts of a joint in response to one type of stimulus but also in the same part of the joint with different stimuli. Surprisingly, therefore, it is widely reported that the proportions of the different inflammatory cells (polymorphs, lymphocytes, and mononuclear cells) in synovial fluid are similar in the various 
clinically defined inflammatory arthropathies; an observation that could be construed as evidence of a single cause for all the inflammatory arthropathies. Superficially this observation is true, but when detailed studies of synovial fluid are undertaken a minority of fluids differ markedly in their cell content from the most commonly encountered pattern and even in the majority subtle differences in the cellular composition of the synovial fluid are usual. This apparently unpredictable behaviour of synovial fluid cells has confounded past attempts to use cytology diagnostically. Perhaps perversely, however, it is the very diversity of cellular infiltrates into synovial fluid that has turned out to be its most important characteristic. Although it might be expected that two similar initiators of inflammation might induce two similar responses, unless the stimuli are identical our understanding of the complex interactions occurring within inflamed tissues would dictate that the cellular responses would not be exactly the same. Therefore if there are differences, no matter how small, in the factors initiating the various inflammatory arthropathies there will be differences in the inflammatory response, though it may be necessary to examine the inflammatory response very carefully to detect the subtle differences present. The relation between the inflammatory cell population in the fluid and the stimulus can also be expressed the other way round. Thus the more divergent the cell populations in samples of synovial fluid the more fundamental the differences between the inflammatory stimuli.

Inflammation, as it is generally perceived, is a term that describes a group of responses by an organism to unwelcome and often potentially harmful stimuli. At the tissue level the distinction between these types of response and other changes consequent upon harmful stimuli, such as trauma, that do not stimulate a classical inflammatory reaction can be difficult to define. All the available evidence would point towards these other responses also being complex interactions between cells and their environment but ones which do not necessarily involve recruitment of classical inflammatory cells. Such events are seen in the 'non-inflammatory' arthropathies. Although the cell-cell and cellmatrix interactions in these responses are much less well studied, there is no reason to believe that the same broad principles that control the relation between initial insult, tissue composition, and cellular response will not also apply in these disorders.

It therefore follows that in both inflammatory and non-inflammatory joint diseases the cellular composition of synovial fluid is dictated by the balance between the complex intra-articular cell-cell and cell-matrix interactions. It also follows that the patterns of cells reflect those interactions and change as they do.

Synovial fluid cytoanalysis is the technique by which the peculiar physical properties of the tissue are exploited to extract cells from the synovial fluid biopsy specimen. By careful examination it is now possible to identify many cell types morphologically in synovial fluid. ${ }^{24}$ Many of these are present in low concentrations, but it is they that show the greatest disease variation. As techniques for probing cells become more sophisticated, ${ }^{25}$ such as immunohistochemistry for identifying cell or function specific epitopes on cell membranes and in situ hybridisation for demonstrating mRNA to specific functional proteins, the true diversity of the synovial fluid cells is being uncovered. It is even possible to dissect the complex cell surface saccharides that represent the informational molecules pivotal to cellular interactions by the combined use of lectin histochemistry and specific saccharidases. Thus the cytological preparation is changing from being simply a medium for the morphological study of synovial fluid cells (although recent studies have shown that this is still a fertile area of investigation $^{24} 26$ ) to one in which fundamental disease mechanisms can be explored. As such it offers an as yet underexploited resource for studying the processes of joint disease.

In theory it should be possible to exploit the relation between synovial fluid cellular reactions and the initiating stimulus as a diagnostic test. In practice the technique does indeed offer the possibility of identifying within the synovial fluid patterns of cells that are of diagnostic and prognostic value. ${ }^{24} 2628-30$ Synovial fluid cytoanalysis is particularly important in certain clinical settings: $(a)$ Where the clinical syndrome is ill defined, as in juvenile arthritis and monoarthropathies and oligoarthropathies of recent onset, the underlying pathogenic mechanisms in the joint often manifest themselves before the clinical syndrome develops. Identification of the underlying disease mechanisms by analysis of synovial fluid may allow specific treatment to be introduced earlier in the course of the disease than would otherwise be possible. (b) Because it can be used to discriminate between diseases that may be clinically similar synovial fluid cytoanalysis is also important as a screening test, particularly for non-specialists such as general medical practitioners, who can use the data generated to decide upon referral policy. (c) As the cell populations reflect the disease processes, in certain circumstances synovial fluid cytoanalysis can be used for titrating complex or potentially hazardous treatment to response. (d) For monitoring disease and disease mechanisms synovial fluid cytoanalysis has the advantage of giving highly specific information about the joint under investigation in a way that serum or urine analysis cannot.

Cytoanalysis also offers completely new ways of thinking about disease mechanisms operating within individual joints and in particular allows arthropathies to be reclassified both within and outside the boundaries of clinical or clinicopathological syndromes. For instance, in this laboratory it has been shown that within the clinical syndrome of seropositive rheumatoid disease patients can be placed into prognostic groups on the basis of synovial fluid cell populations. ${ }^{30}$ Conversely, synovial fluid cytoanalysis shows that the cell populations within joints of patients with ankylosing spondylitis, psoriatic arthritis, reactive arthritis, postinfective arthritis, and the arthritis associated with in- 
flammatory bowel disease are so similar and characteristic that it is highly probable that the disease processes within these disorders are almost identical. ${ }^{28}$

In summary, the essence of this hypothesis is that synovial fluid is a unique connective tissue. It is in direct functional contact with arguably all the most important structures within the diarthrodial joint. In all types of insult changes occur in the quantity and type of cells within the tissue, which reflect the nature of the insult. Closer investigation of the cellular changes within synovial fluid will allow a clearer understanding of the pathogenetic mechanisms operating within diseased joints. Recognition of patterns of disturbance within the cell populations of the joint gives important diagnostic and prognostic information that can be obtained in no other way and will lead to a reclassification of arthritis on the basis of pathogenetic mechanisms rather than present day clinical criteria.

1 Davies D V, ed. Gray's anatomy descriptive and applied. 34th ed. London: Longmans, 1967; 487.

2 Holt P J L. Joint cartilage: physiology and changes in arthritis. In: Holt P J L, ed. Current topics in connective tissue diseases. Edinburgh: Churchill Livingstone, 1975 :
$24-47$.

3 Malemud C J, Moskowitz R W. Physiology of articular cartilage. Clin Rheum Dis 1981; 7: 29-55.

4 Ghadially F N. Fine structure of symovial joints. London: Butterworth, 1983.

5 Hogg N, Palmer D G, Revell P A. Mononuclear phagocytes of normal and rheumatoid synovial membrane identified by monoclonal antibodies. Immunology 1985; 56: 673-81.

6 Linck G, Stocker S, Grimaud J A, Porte A. Distribution of immunoreactive fibronectin and collagen (type I, III, IV) in mouse joints. Histochemistry 1983; 77: 323-7.

7 Scott D L, Salmon M, Morris C J. Laminin and vascular proliferation in rheumatoid arthritis. Ann Rheum Dis 1984; 43: $551-6$.

8 Henderson B, Edwards J C W. The synovial lining in health and disease. London: Chapman and Hall, 1987.

9 Barland P, Novikoff A B, Hamerman D. Electron microscopy of the human synovial membrane. $\mathcal{f}$ Cell Biol 1962; 14: 207-20.
10 Simkin P A. Synovial physiology. In: McCarthy D J, ed Arthritis and allied conditions. Philadelphia: Lea and Arthritis and allied cond

11 Harris E D. Biology of the joint. In: Kelley W N, Harris E D, Ruddy S, Sledge C B, eds. Textbook of rheumatology. Philadelphia: Saunders, 1981: 255-76.

12 Happey F. Studies of the structure of the human intervertebral disc in relation to its functional and aging processes. In: Sokoloff $\mathrm{L}$, ed. The joints and symovial fluid. Vol II. London: Academic Press, 1980: 95-137.

13 McEvedy B V. Simple ganglia. Br f Surg 1962; 49: 585-94. 4 Canosos J J. Bursae, tendons and ligaments. Clin Rheum Dis 1981; 7: 189-221.

15 Maini $R$ N. Immune complexes and complement. In: Scott $\mathrm{J} T$, ed. Copeman's textbook of the rheumatic diseases. J T, ed. Copeman's textbook of the rheumatic dised
Edinburgh: Churchill Livingstone, 1986: 376-410.

16 Simon S R. Biomechanics of joints in: Kelley W N, Harris E D, Ruddy S, Sledge C B, eds. Textbook of rheumatology. E D, Ruddy S, Sledge C B, eds. Textbook

Philadelphia: Saunders, 1981: 294-316.
17 Currey H L F, Vernon-Roberts B. Examination of synovial fluid. Clin Rheum Dis 1976; 2: 149-77.

18 Roy S, Ghadially F N. Pathology of experimental haemarthrosis. Ann Rheum Dis 1966; 25: 402-10.

19 Edwards J C W, Willoughby D A. Demonstration of bone marrow derived cells in synovial lining using giant lysosomal granules as genetic markers. Ann Rheum Dis 1982; 41: $282-6$.

20 Ropes M, Bauer W. Synovial fluid changes in joint disease. Cambridge, Mass: Harvard University Press, 1953.

21 Schumacher H R, Kitridou R C. Synovitis of recent onset: a clinicopathological study during the first month of disease. Arthritis Rheum 1972; 15: 465-72.

22 Wolf A W, Benson D R, Shiji J, et al. Current concepts in synovial fluid analysis. Clin Orthop 1978; 134: 261-5.

23 Zvaifler N J. The immunopathology of joint inflammation in rheumatoid arthritis. Advan Immunol 1973; 16: 265-336.

24 Freemont A J, Denton J, Chuck A, Holt P J L, Davies M J. Diagnostic value of synovial fluid microscopy: a reassessment and rationalisation. Ann Rheum Dis 1991; 50: 101-7.

25 Freemont $A$ J. The use of the diagnostic tissue biopsy in rheumatology. ARC topical review. London: Arthritis and Rheumatism Council, 1989: No 13.

26 Freemont A J, Denton J. Synovial fluid findings early in traumatic arthritis. $\mathcal{F}$ Rheumatol 1988; 15: 881-2.

27 Freemont A J, Denton J. Diagnostic applications of synovial fluid cytoanalysis. 7 Pathol 1987; 151: 29 A.

28 Freemont A J, Denton J. The disease distribution of synovial fluid mast cells and cytophagocytic mononuclear cells in inflammatory arthritis. Ann Rheum Dis 1985; 44: 312-5.

29 Freemont A J. Pathology of ankylosing spondylitis. In: Calabro D C, Dick W C, eds. New clinical applicationsrheumatology-ankylosing spondylitis. Lancaster: MTP Press, 1988: 1-22.

30 Davies M J, Denton J, Freemont A J, Holt P J L. Comparison of serial synovial fluid cytology in rheumatoid arthritis: delineation of subgroups with prognostic implications. Ann Rheum Dis 1988; 47: 559-62. 